\title{
Characterization of Extended Width Optical Dipole Antennas
}

\author{
Tae Joon Seok, Arash Jamshidi, Amit Lakhani, Kyoungsik Yu, Hyuck Choo, Owen Miller, \\ Eli Yablonovitch, and Ming C. Wu \\ Department of Electrical Engineering and Computer Sciences, University of California, Berkeley, CA 94720, USA \\ Contacte-mail: tjseok@eecs.berkeley.edu
}

\begin{abstract}
Optical dipole antennas with varying length and width are fabricated using e-beam lithography. Antennas with wider width are shown to exhibit stronger scattering while preserving the same resonance frequency.

(C)2010 Optical Society of America

OCIS codes: (240.6695) Surface-enhanced Raman scattering; (350.4238) Nanophotonics and photonic crystals
\end{abstract}

\section{Introduction}

Optical antennas offer interesting solutions in a variety of applications including biochemical sensing, imaging, data storage, wavelength conversion, and energy harvesting [1]. The ability to focus the light beyond the diffraction limit with optical antenna is especially important in improving the sensitivity of optical sensing techniques such as fluorescent detection [2] and surface-enhanced Raman spectroscopy (SERS) [3]. For example, bowtie optical antenna is employed to confine the incident light in the tight focal volume of the optical antenna, and the focused electromagnetic wave interacts with analyte molecules within the small focal volume [1, 2, 4]. However, in typical sensing applications, analytes are adsorbed uniformly on the substrates surface, and most analyte molecules lie outside the optical antenna's focal volume because of low aerial density of the hot spots. In this paper, we investigate the effect of antenna width variation on the antenna resonance wavelength. By measuring the optical scattering spectra from antenna arrays, we found that wider antennas exhibit much stronger scattering intensity while maintaining the same resonance frequency. By increasing the antenna width, we can effectively increase the hot spot volume which is important for SERS and other sensing applications.

\section{Optical Antenna Design and Fabrication}

Optical dipole antenna arrays with various lengths $(220,280,340$, and $400 \mathrm{~nm})$ and widths $(70,100,130,150$, and $180 \mathrm{~nm}$ ) are fabricated on an indium-tin-oxide (ITO)-coated glass substrate using high-resolution electron beam lithography and lift-off technique. A 3-nm germanium layer is evaporated as an adhesion layer, and followed by 30 $\mathrm{nm}$ gold evaporation. The Ge seed layer reduces the roughness of the gold surface from 3 to $1 \mathrm{~nm}$, similar to the silver results in [5]. Each array contains identical dipole antennas with a 1- $\mu \mathrm{m}$ pitch. Figure 1(a) and (b) show examples of the patterned dipole antenna arrays and individual antenna with different dimensions. According to our scanning electron microscope (SEM) measurements, antennas have $\sim 16 \mathrm{~nm}$ wide feed gaps.

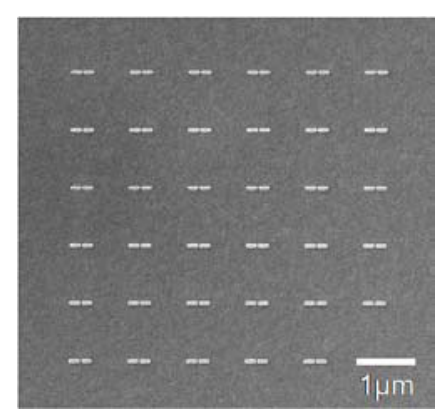

(a)

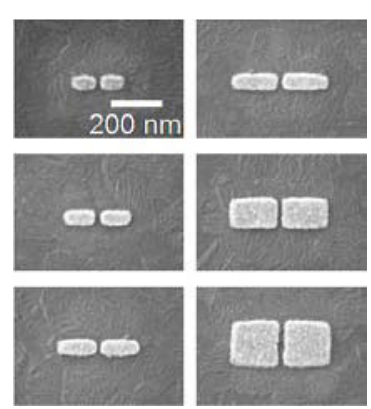

(b)

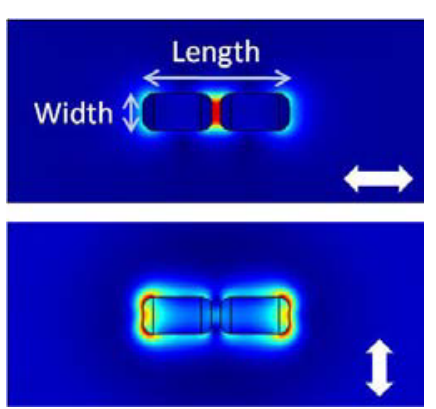

(c)

Fig. 1 (a) SEM of an optical dipole antenna array. Identical dipole antennas are equally distributed with a 1- $\mu \mathrm{m}$ pitch in one array. (b) Magnified SEMs of individual optical dipole antennas. Left and right column representatively show the dimension variation in antenna length and width, respectively. Each antenna has $\sim 16 \mathrm{~nm}$ gap separation. (c) Electric field magnitude distribution of the dipole antennas excited by linearly polarized plane waves. White arrows represent the polarization of the plane wave, incident from the surface normal direction.

To understand the resonant coupling to optical antennas, we simulate antenna arrays using a finite element method (FEM)-based computer software. Dipole antenna arrays with a $1-\mu \mathrm{m}$ pitch are modeled with a periodic boundary condition and excited by plane waves from the top. Figure 1(c) shows typical electric field profiles at resonance with two different polarizations. The electric field is greatly enhanced only when the input polarization 


\section{CFI4.pdf}

is aligned with the antenna optical axis. Our simulation also shows that the antenna resonance mainly depends on the antenna length.

\section{Experiments and Results}

Optical scattering from the antenna arrays are measured under broadband dark field illumination to characterize the antenna resonance in the visible and near-infrared wavelength ranges. An inverted microscope with a 40x objective (Nikon TE2000) and a spectrometer are used to collect and analyze the scattered light from the optical antennas.

Figure 2(a) shows the measured and simulated scattering spectra for antennas with $220 \mathrm{~nm}$ and $280 \mathrm{~nm}$ length. Simulated scattering spectra are obtained by subtracting absorption cross sections of antennas from their extinction cross sections. $220 \mathrm{~nm}$ long antennas show measured resonance peaks at $763 \mathrm{~nm}$ and $280 \mathrm{~nm}$ long antennas show resonance peaks at $846 \mathrm{~nm}$, which are red-shifted by $83 \mathrm{~nm}$ relative to the resonance peaks of $220 \mathrm{~nm}$ long antennas. Simulation results show similar trends but the resonance peak values are slightly red-shifted relative to the measured values. This is caused by the actual sample having an ITO-coated glass substrate which has a refractive index slightly smaller than ITO. The substrate used for simulations is only an ITO layer with a higher refractive index, resulting in a red-shift in the resonance peak values.

Figure 2(b) shows the resonance wavelengths for $220 \mathrm{~nm}$ (black) and $280 \mathrm{~nm}$ (red) long antennas with various antenna widths. Varying antenna width does not result in a considerable shift of resonance peak since kinetic inductance of antennas is not dominant in this large width regime. Individual scattering spectra of $220 \mathrm{~nm}$ and 280 $\mathrm{nm}$ long antennas are shown in Figure 2(c) and (d), respectively. Dipole antennas with wider width exhibits stronger scattering signal. The wider width also produces an extended hot spot that can greatly increase the surfaceenhanced Raman spectroscopy (SERS) signal.

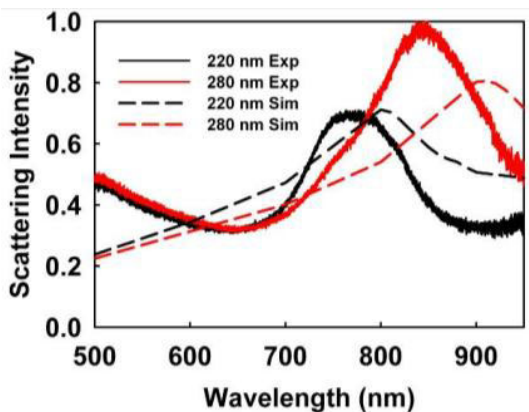

(a)

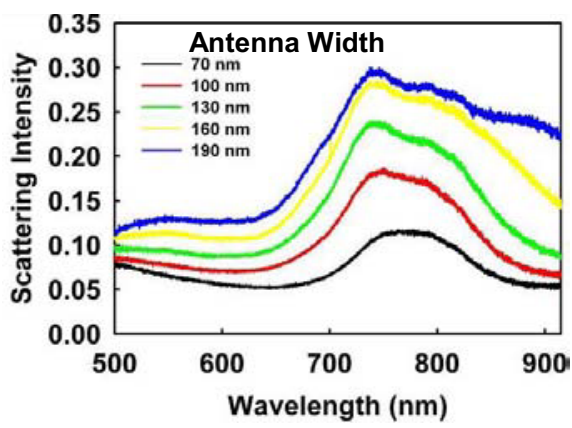

(c)

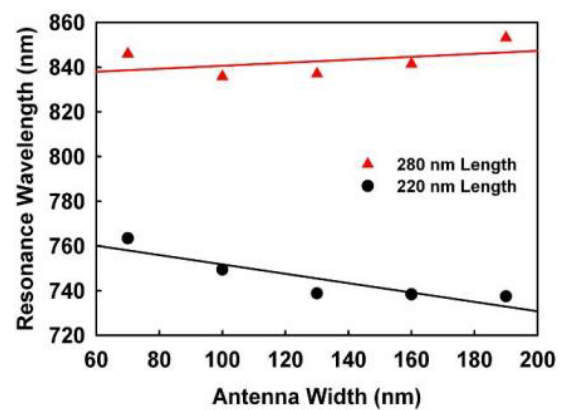

(b)

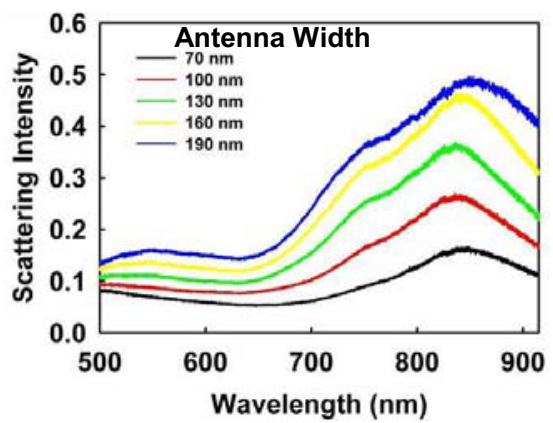

(d)

Fig. 2 (a) Experimental (solid line) and simulated (dashed line) scattering spectra of dipole antennas with $60 \mathrm{~nm}$ width and $220 \mathrm{~nm}$ length (black curves) and $280 \mathrm{~nm}$ length (red curves). (b) Measured antenna resonance wavelength as a function of antenna width. Black and red dots represent the antenna length of $220 \mathrm{~nm}$ and $280 \mathrm{~nm}$, respectively. (c) Measured antenna scattering spectra for $220 \mathrm{~nm}$ length and variable widths. (d) Measured antenna scattering spectra for $280 \mathrm{~nm}$ length and variable widths.

\section{References}

[1] P. Bharadwaj, B. Deutsch, and L. Novotny, “Optical antennas,” Advances in Optics and Photonics 1, 438-483 (2009).

[2] A. Kinkhabwala, Z. Yu, S. Fan, Y. Avlasevich, K. Mullen, and W.E. Moerner, "Large single-molecule fluorescence enhancements produced by a bowtie antenna," Nature Photonics 3, 654-657 (2009).

[3] D. P. Fromm, A. Kinkhabwala, P.J. Schuck, and W.E. Moerner, "Exploring the chemical enhancement for surface-enhanced Raman scattering with Au bowtie nanoantennas," J. Chem. Phys. 124, 061101 (2006).

[4] P.J. Schuck, D.P. Fromm, A. Sundaramurthy, G.S. Kino, and W.E. Moerner, "Improving the mismatch between light and nanoscale objects with gold bowtie nanoantennas," Phys. Rev. Lett. 94, 017402 (2005).

[5] V.J. Logeeswaran, et al., "Ultrasmooth silver thin film deposited with a Germanium nuclear layer," Nano Lett. 9, 178-182 (2009). 